Bull. Austral. Math. Soc.

Vol. 45 (1992) [37-41]

\title{
A FORMULA ON THE APPROXIMATE SUBDIFFERENTIAL OF THE DIFFERENCE OF CONVEX FUNCTIONS
}

\author{
J.E. Martínez-Legaz and A. Seeger
}

We give a formula on the $\varepsilon$-subdifferential of the difference of two convex functions. As a by-product of this formula, one recovers a recent result of Hiriart-Urruty, namely, a necessary and sufficient condition for global optimality in nonconvex optimisation.

\section{The $\varepsilon$-SUbdifferential of A DC-FunCtion}

Whether the extended-real-valued function $f: X \longrightarrow \overline{\mathbb{R}}$ is convex or not, we use the standard expression

$$
\partial_{\varepsilon} f\left(x_{0}\right):=\left\{u \in X^{*}: f(x) \geqslant f\left(x_{0}\right)+\left\langle u, x-x_{0}\right\rangle-\varepsilon \text { for all } x \in X\right\}
$$

as definition for the $\varepsilon$-subdifferential of $f$ at $x_{0} \in X$. Here $X$ and $X^{*}$ are locally convex (real) topological linear spaces paired in duality by a bilinear form $\langle\cdot, \cdot\rangle: X^{*} \times$ $X \longrightarrow \mathbb{R}$. As it is customary, we assume that $\varepsilon$ is a nonnegative real number and that $x_{0}$ is a point at which $f$ is finite. The particular instance $\varepsilon=0$ corresponds, of course, to the usual subdifferential

$$
\partial f\left(x_{0}\right):=\left\{u \in X^{*}: f(x) \geqslant f\left(x_{0}\right)+\left\langle u, x-x_{0}\right\rangle \text { for all } x \in X\right\} .
$$

It is important to note that in the nonconvex case, the $\varepsilon$-subdifferential mapping $\partial_{\varepsilon} f: X \rightrightarrows X^{*}$ may be empty-valued at some points.

Formulas for evaluating the $\varepsilon$-subdifferential of a convex function can be found, for instance, in Kutateladze [4] and Hiriart-Urruty [1]. These authors established calculus rules for most of the operations preserving convexity (like addition, inf-convolution, upper envelope, et cetera). They did not consider, however, the case of the subtraction, an operation which does not preserve the convexity in general.

The purpose of this note is to write a formula on the $\varepsilon$-subdifferential of a DCfunction, that is, of a function $f$ which can be represented as the difference

$$
x \in X \longrightarrow f(x):=g(x)-h(x)
$$

\section{Received 8th January 1991}

The first author acknowledges financial support from the Dirección General de Investigación Científica y Técnica (DGICYT), under project PS89-0058.

Copyright Clearance Centre, Inc. Serial-fee code: 0004-9729/92 \$A2.00+0.00. 
of two convex functions $g, h: X \longrightarrow \mathbb{R} \cup\{+\infty\}$. Since $g$ and $h$ may take the value $+\infty$ at the same time, we adopt here the rule $(+\infty)-(+\infty)=+\infty$. The class of DC-functions has received a great deal of attention in recent time. For a survey on this topic, one may consult Hiriart-Urruty [2]. In particular, one can find there a formula on the Fenchel conjugate of the difference of two convex functions. To the best of our knowledge, a formula on the $\varepsilon$-subdifferential of such type of difference has not been established yet.

In next theorem,

$$
A^{*} B=\left\{u \in X^{*}: u+B \subset A\right\}
$$

stands for the "star-difference" between two sets $A$ and $B$ in $X^{*}$ (see $[2$, p.56]).

Theorem 1. Let $g, h: X \longrightarrow \mathbb{R} \cup\{+\infty\}$ be two lower-semicontinuous proper convex functions, finite at $x_{0} \in X$. Then, for every $\varepsilon \geqslant 0$, one has

$$
\partial_{\varepsilon}(g-h)\left(x_{0}\right)=\bigcap_{\lambda \geqslant 0}\left\{\partial_{\varepsilon+\lambda} g\left(x_{0}\right) * \partial_{\lambda} h\left(x_{0}\right)\right\} .
$$

Setting $\varepsilon=0$, one gets in particular

$$
\partial(g-h)\left(x_{0}\right)=\bigcap_{\lambda \geqslant 0}\left\{\partial_{\lambda} g\left(x_{0}\right)^{*} \partial_{\lambda} h\left(x_{0}\right)\right\} .
$$

Proof: By definition, $u \in \partial_{\varepsilon}(g-h)\left(x_{0}\right)$ if and only if

$$
(g-h)(x) \geqslant(g-h)\left(x_{0}\right)+\left\langle u, x-x_{0}\right\rangle-\varepsilon \quad \text { for all } \quad x \in X
$$

or, what is equivalent,

$$
g(x)-h_{u}(x) \geqslant g\left(x_{0}\right)-h_{u}\left(x_{0}\right)-\varepsilon \quad \text { for all } \quad x \in X
$$

where $h_{u}:=h+\langle u, \cdot\rangle$. But, according to the Toland-Singer duality theorem $[6,7]$, one can write

$$
\inf _{x \in X}\left\{g(x)-h_{u}(x)\right\}=\inf _{y \in X^{*}}\left\{\left(h_{u}\right)^{*}(y)-g^{*}(y)\right\}
$$

where the convention $(+\infty)-(+\infty)=\infty$ applies on both sides of this equality, and $\varphi^{*}: X^{*} \longrightarrow \mathbb{R} \cup\{+\infty\}$ stands for the Fenchel conjugate of $\varphi: X \longrightarrow \mathbb{R} \cup\{+\infty\}$. Consequently, (5) is equivalent to

$$
\left(h_{u}\right)^{*}(y)-g^{*}(y) \geqslant g\left(x_{0}\right)-h_{u}\left(x_{0}\right)-\varepsilon \text { for all } y \in X^{*} \text {. }
$$


Now, introduce the notation

$$
\begin{aligned}
& p(y):=\left(h_{u}\right)^{*}(y)+h_{u}\left(x_{0}\right)-\left\langle y, x_{0}\right\rangle \\
& q(y):=g^{*}(y)+g\left(x_{0}\right)-\left\langle y, x_{0}\right\rangle
\end{aligned}
$$

and write (7) in the form

$$
p(y) \geqslant q(y)-\varepsilon \text { for all } y \in X^{*} .
$$

The inequality (8) relating the nonnegative functions $p$ and $q$ can be expressed in terms of an inclusion

$$
\left\{y \in X^{*}: p(y) \leqslant \lambda\right\} \subset\left\{y \in X^{*}: q(y) \leqslant \varepsilon+\lambda\right\} \text { for all } \lambda \geqslant 0
$$

between their corresponding level sets. But, from the very definition of $p$ and $q$, one sees that

$$
\left\{y \in X^{*}: p(y) \leqslant \lambda\right\}=\partial_{\lambda} h_{u}\left(x_{0}\right)=u+\partial_{\lambda} h\left(x_{0}\right),
$$

and

$$
\left\{y \in X^{*}: q(y) \leqslant \varepsilon+\lambda\right\}=\partial_{e+\lambda} g\left(x_{0}\right) .
$$

Summarising, one has proved that $u \in \partial_{\varepsilon}(g-h)\left(x_{0}\right)$ if and only if

$$
u+\partial_{\lambda} h\left(x_{0}\right) \subset \partial_{\varepsilon+\lambda} g\left(x_{0}\right) \text { for all } \lambda \geqslant 0 .
$$

This is precisely what formula (3) says.

REMARK 2. The lower-semicontinuity and the convexity of $g$ are not essential assumptions in Theorem 1. In fact, these assumptions have been used only for writing equality (6). It is known that formula (6) is still valid if $g: X \rightarrow \mathbb{R} \cup\{+\infty\}$ is arbitrary. On the other hand, formula (6) has been extended to the more general conjugation framework of Moreau. Consequently, a formula similar to (3) can be obtained for the corresponding generalised concept of $\varepsilon$-subdifferential. For the above mentioned extensions of formula (6), see for instance Martínez-Legaz [5, Theorem 3.1].

\section{AN APPLICATION TO DC-PROGRAMMING}

The different consequences and applications of Theorem 1 will not be explored in this short note. We shall mention, however, the application we had in mind when we established formula (3). Recall that a point $x_{0} \in X$ is said to be an $\varepsilon$-minimum of the function $f: X \longrightarrow \overline{\mathbb{R}}$, if $f\left(x_{0}\right)$ is finite and

$$
f\left(x_{0}\right)-\varepsilon \leqslant f(x) \text { for all } x \in X .
$$

As an illustration on the use of Theorem 1, we exhibit a necessary and sufficient condition for $\varepsilon$-minimality due to Hiriart-Urruty [3]. 
Corollary 3. (see [3, Theorem 4.4]) Let $g$ and $h$ be as in Theorem 1. A necessary and sufficient condition for $x_{0} \in X$ be an $\varepsilon$-minimum of $x \in X \mapsto f(x):=$ $g(x)-h(x)$ is that

$$
\partial_{\lambda} h\left(x_{0}\right) \subset \partial_{e+\lambda} g\left(x_{0}\right) \text { for all } \lambda \geqslant 0
$$

In particular, $x_{0} \in X$ is a global minimum of $f=g-h$ if and only if

$$
\partial_{\lambda} h\left(x_{0}\right) \subset \partial_{\lambda} g\left(x_{0}\right) \quad \text { for all } \lambda \geqslant 0
$$

Proof: Condition (11) is equivalent to $0 \in \partial_{e}(g-h)\left(x_{0}\right)$.

REMARK 4. Theorem 1 can, in turn, be derived from Corollary 3. Indeed, starting from (5) it suffices to apply the optimality condition (11) to the $\varepsilon$-minimum $x_{0}$ of the function $g-h_{u}$. In this way, one gets

$$
\partial_{\lambda} h_{u}\left(x_{0}\right) \subset \partial_{e+\lambda} g\left(x_{0}\right) \text { for all } \lambda \geqslant 0 \text {, }
$$

which is equivalent to (10). The proof we gave in Section 1 was inspired on a proof of (12) due to Pham Dinh Tao, communicated to us by Hiriart-Urruty (personal communication).

\section{References}

[1] J.-B. Hiriart-Urruty, ' $\varepsilon$-subdifferential calculus', in Convex analysis and optimization: Research Notes in Mathematics 57, pp. 43-92 (Pitman Publishers, 1982).

[2] J.-B. Hiriart-Urruty, 'Generalized differentiability, duality and optimization for problems dealing with differences of convex functions', in Converity and duality in optimization: Lecture Notes in Econom. and Math. Systems 256, pp. 37-70 (Springer-Verlag, Berlin, Heidelberg, New York, 1986).

[3] J.-B. Hiriart-Urruty, 'From convex optimization to nonconvex optimization', in Nonsmooth optimization and related topics, Editors F.H. Clarke, V.F. Demyanov, F.Giannessi, pp. 219-239 (Plenum Press, 1989).

[4] S. S. Kutateladze, 'Convex e-programming', Soviet Math. Dokl. 20 (1979), 391-393.

[5] J.-E. Martínez-Legaz, 'Generalized conjugation and related topics', in Generalized convexity and fractional programming with economic applications: Lect. Notes in Econom. and Math. Systems 345, pp. 198-218 (Springer-Verlag, Berlin, Heidelberg, New York, 1990).

[6] I. Singer, 'A Fenchel-Rockafellar type duality theorem for maximization', Bull. Austral. Math. Soc. 29 (1979), 193-198.

[7] J. Toland, 'A duality principle for nonconvex optimization and the calculus of variations', Arch. Rational Mech. Anal. 71 (1979), 41-61. 
Dept. d'Economia i d'Història Econòmica Universitat Autònoma de Barcelona 08193-Bellaterra, Barcelona

Spain

and

Dept. de Matemàtica Aplicada i Anàlisi Universitat de Barcelona

08071 - Barcelona

Spain
Dept. de Matemàtica Aplicada i Anàlisi Universitat de Barcelona 08071 - Barcelona

Spain 